\title{
Construction and characterization of recombinant adenovirus carrying a mouse TIGIT-GFP gene
}

\author{
J.M. Zhengi" ${ }^{1 *}$, J.L. Cui ${ }^{2 *}$, W.T. He ${ }^{3}$, D.W. $\mathrm{Yu}^{4}$, Y. Gao ${ }^{4}$, L. Wang ${ }^{4}$, Z.K. Chen ${ }^{4}$ and \\ H.M. Zhou ${ }^{5}$ \\ 'Department of Cardio-Thoracic Surgery, Zhongshan City People Hospital, \\ Zhongshan, Guangdong, China \\ ${ }^{2}$ Department of Cardio-Thoracic Surgery, \\ Hospital of Traditional Chinese Medicine of Zhongshan, Guangdong, China \\ ${ }^{3}$ Department of Endocrinology, Tongji Hospital, Tongji Medical College, \\ Hua zhong University of Science and Technology, Wuhan, China \\ ${ }^{4}$ Institute of Organ Transplantation, Tongji Hospital, Tongji Medical College, \\ Huazhong University of Science and Technology, \\ Key Laboratory of Ministry of Health, Key Laboratory of Ministry of Education, \\ Wuhan, China \\ ${ }^{5}$ Department of Cardio-Thoracic Surgery, Tongji Hospital, Tongji Medical College, \\ Hua zhong University of Science and Technology, Wuhan, China \\ ${ }^{*}$ These authors equally contributed to this study. \\ Corresponding authors: H.M. Zhou / Z.K. Chen \\ E-mail: amzhmin@126.com / zc104@medmail.com.cn
}

Genet. Mol. Res. 14 (4): 18650-18661 (2015)

Received August 15, 2015

Accepted October 8, 2015

Published December 28, 2015

DOI http://dx.doi.org/10.4238/2015.December.28.14

ABSTRACT. Recombinant adenovirus vector systems have been used extensively in protein research and gene therapy. However, the construction and characterization of recombinant adenovirus is a tedious and timeconsuming process. TIGIT is a recently discovered immunosuppressive molecule that plays an important role in maintaining immunological balance. The construction of recombinant adenovirus mediating TIGIT expression 
must be simplified to facilitate its use in the study of TIGIT. In this study, the TIGIT gene was combined with green fluorescent protein (GFP); the TIGIT-GFP gene was inserted into a gateway plasmid to construct a TIGITGFP adenovirus. HEK 293A cells were infected with the adenovirus, which was then purified and subjected to virus titering. TIGIT-GFP adenovirus was characterized by flow cytometry and immunofluorescence, and its expression in mouse liver was detected by infection through caudal vein injection. The results showed the successful construction of the TIGITGFP adenovirus (5 x $10^{10} \mathrm{PFU} / \mathrm{mL}$ ). Co-expression of TIGIT and GFP was identified in 293A and liver cells; synthesis and positioning of TIGITGFP was viewed under a fluorescence microscope. TIGIT-GFP was highly expressed on liver cells 1 day (25.53\%) after infection and faded 3 days (11.36\%) after injection. In conclusion, the fusion of TIGIT with GFP allows easy, rapid, and uncomplicated detection of TIGIT translation. The construction of a TIGIT-GFP adenovirus, mediating TIGIT expression in vitro and in vivo, lays the foundation for further research into TIGIT function and gene therapy. Moreover, the TIGIT-GFP adenovirus is a helpful tool for studying other proteins (which could replace the TIGIT gene).

Key words: Recombinant adenovirus vector; TIGIT; X-GFP fusion gene; Mouse; Immunesuppression

\section{INTRODUCTION}

Adenovirus vector systems have been used extensively in protein research and gene therapy, among other applications (Lewis et al., 2014; Wang et al., 2014). Adenovirus vectors are capable of infecting a broad range of mammalian cells for stable expression of recombinant proteins, which could assist in subsequent analyses (Ma et al., 2010; Wei et al., 2010). Tracing proteins, such as green fluorescent proteins (GFP), are widely used in the tracing of protein synthesis, position, and metabolism, without identifying the proteins using antibodies (Wang et al., 2011). X-GFP fusion proteins can be created artificially by genetic engineering, facilitating future biological research (Li et al., 1999; Wong et al., 2011; Yang et al., 2011). Therefore, the construction and application of X-GFP-ADV (adenovirus) could be an efficient method for the in vitro and in vivo study of protein function in a series of mammalian cells.

TIGIT was recently discovered as an inhibitory molecule that attenuates the T-cell response in the presence of dendritic cells (DCs) via interleukin-10 (IL-10) secretion in vitro (Yu et al., 2009). The interaction of TIGIT with PVR inhibits human NK cell cytotoxicity in vivo (Stanietsky et al., 2009), and soluble TIGIT inhibits the development of autoimmune diseases in mice (Joller et al., 2011). In addition, TIGIT-deficient mice are more susceptible to autoimmune challenge (Levin et al., 2011). In human trials, the TIGIT signal was found to inhibit T cell proliferation and cytokine production (Lozano et al., 2012). These studies show that TIGIT plays an important role in immune suppression.

In this study, the TIGIT sequence was cloned from mouse spleen by reverse transcription polymerase chain reaction PCR. Primers used for TIGIT cloning and genetic recombination were designed as required. The TIGIT gene was cloned and introduced into the pEGFP-N1 plasmid to form a TIGIT-GFP segment, which was cut and inserted into a gateway plasmid. TIGIT-GFPADV was constructed after LR recombination. TIGIT-GFP-ADV was introduced into HEK 293A 
cells, amplified, purified, and finally subjected to virus tittering. The amplification and virus tittering of TIGIT-GFP-ADV was observed under a fluorescence microscope via the expression of the GFP tag. Subsequently, 293A cells infected with TIGIT-GFP-ADV were detected by flow cytometry and immunofluorescence to confirm the co-expression of TIGIT and GFP. For in vivo analyses, C57BL/6 mice were infected with TIGIT-GFP-ADV, and the liver was harvested for flow cytometry and immunofluorescence. Our results showed the successful construction of the TIGIT-GFP-ADV. This mediated the TIGIT expression in vitro and in vivo, which lay the foundation of further research of TIGIT function and gene therapy.

\section{MATERIAL AND METHODS}

\section{Reverse transcription PCR of TIGIT from mouse}

Total RNA was isolated from the organs of C57BL/6 mice using TRIzol ${ }^{\circledR}$ reagent (Invitrogen, Carlsbad, CA, USA). The first cDNA was synthesized using a ReverTra Ace - $\alpha$ - kit (Toyobo, OSAKA, JAPAN) according to the manufacturer protocols. The coding sequence of mouse-TIGIT was cloned from the total cDNA by PCR, using the following primers: sense primer: 5'-GCCAGTTT CAGTTGGAGGAGAG-3', anti-sense primer: 5'-CTCGAGAGGGATAGAGAGCTGTCGTTAG-3'. The PCR cycling conditions were set as follows: initial denaturation at $95^{\circ} \mathrm{C}$ for 5 min followed by 30 cycles of $95^{\circ} \mathrm{C}$ for $30 \mathrm{~s}, 58^{\circ} \mathrm{C}$ for $45 \mathrm{~s}$, and $72^{\circ} \mathrm{C}$ for $1 \mathrm{~min}$, and a final extension at $72^{\circ} \mathrm{C}$ for 10 min. A portion of the liquid from each tube was dyed with SYBR green I (Shinegene, Shanghai, China) and observed under UV light after a nucleic acid gel electrophoresis.

Gene cloning and construction of TIGIT-GFP-ADV. The TIGIT sequence was inserted into the multiple cloning sites of pGM-T, using a pGM-T clone kit ( TIANGEN BIOTECH, Beijing, China). TIGIT-pGM-T was transformed into TOP10 Escherichia coli (TIANGEN ) for amplification. The TIG-

IT in pGM-T was cloned by PCR, using a sense (5'-CGGAATTCAGCATGCATGGCTGGCT-3'; underlined bases encode the EcoRI site) and anti-sense 5'-CGGGATCCCCAGTCTTCGATACAGCA ATGA-3'; underlined bases encode the BamHI site) primer, using the conditions described above. The TIGIT sequence was then digested by EcoRI and BamHI, and inserted into pEGFP-N1 using a T4 DNA Ligase (Promega, Madison, WI, USA) to construct the TIGIT-GFP segment. TIGIT-GFP in the pEGFP-N1 was digested using a similar process by EcoRI and Notl, and inserted into pENTR1A. The TIGIT-GFP-pENTR1A was subsequently digested with EcoRI and Notl for identification, and sent to Invitrogen for sequencing.

An LR recombination reaction was performed to fix the TIGIT-GFP-ADV plasmid, using the Gateway LR Clonase II Enzyme Mix (Invitrogen). The TIGIT-GFP-ADV plasmid was then transformed into TOP10 Escherichia coli (Tiangen) to screen for the positive clones. The TIGIT-GFPADV plasmid was then extracted from the bacteria after amplification. Eventually, TIGIT-GFP-ADV was linearized using Pac I (Toyobo).

\section{Culture of 293A cells and generation of TIGIT-GFP-ADV}

The 293A cells were cultured in Dulbecco's modified Eagle's medium (DMEM) containing glucose $(4.5 \mathrm{~g} / \mathrm{L})$, penicillin $(200 \mathrm{U} / \mathrm{mL})$, streptomycin $(100 \mu \mathrm{g} / \mathrm{mL})$, and $10 \%$ fetal bovine serum (FBS). The cells were incubated at $37^{\circ} \mathrm{C}$ in a $5 \% \mathrm{CO}_{2}$ incubator (MCO-18AIC, Sanyo, Japan). Subsequently, $1 \times 10^{6}$ cells were planted in the culture plate. After $24 \mathrm{~h}$, the TIGIT-GFP-ADV was transfected into the cells using Lipofectamine 2000 (Invitrogen) according to the manufacturer 
instructions. The cells expressing TIGIT-GFP were detected after 7-10 days under a fluorescence microscope. The cells were harvested and subjected to three freeze-thaw cycles at $-80^{\circ}$ and $37^{\circ} \mathrm{C}$ to release the virus particles from the 293A cells for further upscaling. The virus particles were purified using a double cesium chloride gradient, as detailed in the manual of the AdEasy Vector System (Qbiogene, Carlsbad, CA , USA). The viral liquid was moved to double CsCl gradient liquid (in a centrifuge tube) containing $4 \mathrm{~mL} \mathrm{CsCl}(1.4 \mathrm{~g} / \mathrm{L})$ and $3 \mathrm{~mL} \mathrm{CsCl}(1.2 \mathrm{~g} / \mathrm{L})$. A blue band could be seen in the tube after centrifugation at $350000 \mathrm{~g}$ at $10^{\circ} \mathrm{C}$ for $2 \mathrm{~h}$. The blue band was extracted into another double $\mathrm{CsCl}$ gradient tube for centrifugation under the same conditions. The blue band was dialyzed to remove the trace $\mathrm{CsCl}$. Finally, the viral liquid was diluted to $10 \%$ in glycerinum and glycerine, and preserved at $-80^{\circ} \mathrm{C}$.

\section{Titration of virus particles}

The virus concentrate was diluted from $10^{-2}$ to $10^{-7}$. Next, 293A cells $\left(5 \times 10^{5}\right)$ in $1 \mathrm{~mL}$ culture solution were seeded to each well of a 12-well plate; subsequently, $0.1 \mathrm{~mL}$ of each dilution was added to each well after $24 \mathrm{~h}$. The cells were observed under a fluorescence microscope after culturing for $48 \mathrm{~h}$. Cells with fluorescence on a $1 \mathrm{~mm}^{2}$ area were counted (for 20 views) and the average was calculated; the presence of $30-50$ cells on each view was considered to be a moderate amount. The titration was calculated using the following formula, as described by Sun et al. (2011). GFP-ADV was similarly constructed as the control group.

$\operatorname{Titer}(\mathrm{PFU} / \mathrm{ml})=[($ Green Fluorescent cells/field $) \times($ fields/well $)] /[$ volume virus $(\mathrm{ml}) \times($ dilution factor $)]$

\section{Immunofluorescence analyses}

The 293A cells were plated on Petri dishes and infected with either TIGIT-GFP-ADV or GFP-ADV at a multiplicity of infection (MOI) of 50 for $24 \mathrm{~h}$. The cells were fixed with ice-cold $80 \%$ methanol for $20 \mathrm{~min}$ and blocked with $1 \%$ bovine serum albumin in phosphate buffered saline (BSA/PBS) for $30 \mathrm{~min}$ at room temperature. The samples were then treated with anti-mouse TIGIT Alexa Fluor 647 (eBioscience, San Diego, CA, USA) diluted in PBS (1:100) and DAPI (100 mg/ $\mathrm{mL})$ diluted in PBS (1:1000). The mixtures were added to the cells and incubated for $30 \mathrm{~min}$. The samples were visualized with a confocal microscope.

\section{Flow cytometry}

The cells were stained with $0.1 \%$ sodium azide and $2 \%$ BSA in PBS for 30 min at room temperature. The cells were then incubated with antibodies from eBioscience (anti-mouse TIGIT). The samples were acquired on a FACS Calibur (BD Biosciences) and analysed using the WinMDI 2.9 software.

\section{Virus infection and detection of mouse liver cells}

Virus particles ( $\left.5 \times 10^{9} \mathrm{PFU}\right)$ were injected into C57BL/6 mice through the caudal vein. The liver was harvested on days $1,3,5$, and 7 . A part of the tissue was frozen at $-80^{\circ} \mathrm{C}$ to obtain the 
frozen section, and subsequently detected by immunofluorescence analysis. The rest were cut into small pieces and ground through a $70 \mu \mathrm{m}$ nylon cell strainer. The cells in the filtrate were washed thrice with PBS and stained for flow cytometry.

\section{RESULTS}

\section{Distribution of TIGIT in mice}

Mouse TIGIT sequences were obtained from the National Center for Biotechnology Information database, and the coding sequence of TIGIT mRNA (Gene ID: 100043314) was confirmed to be from 98 to 823 (726 bp), coding for 241 aa. Structural analyses (UniProt: http://pir.uniprot. org/) indicated that TIGIT contained four sections: a signal peptide (1 to 26 aa) for the membrane location, an extracellular segment (27 to 141 aa) for an immunoglobulin variable domain, and a transmembrane domain (142 to 162 aa) and an intracellular segment (163 to 241 aa) containing an immunoreceptor tyrosine-based inhibitory motif (ITIM). Therefore, we speculated that TIGIT was expressed on the cell surface, and functions by interacting with other cells (Figure 1).
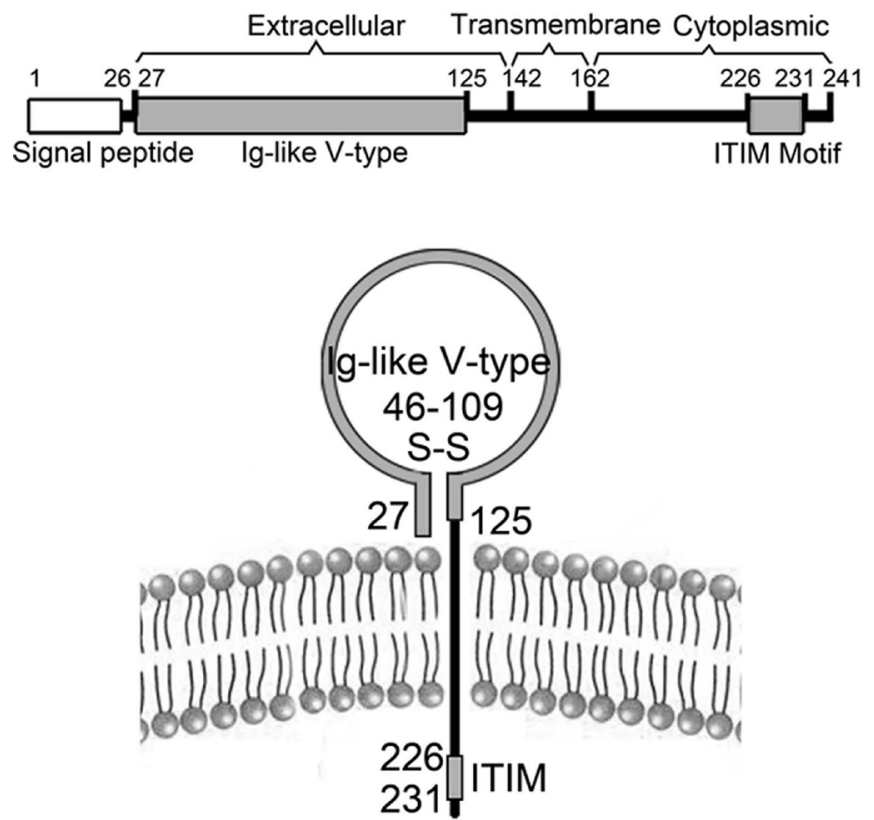

Figure 1. Model for amino acid sequence and spatial structure of TIGIT. Structural analysis indicated that TIGIT contains four sections: a signal peptide (1-26 aa) for membrane location, an extracellular segment (27-141 aa) for an immunoglobulin variable domain, a transmembrane domain (142ᄀ162 aa), and an intracellular segment (163-241 aa) containing an ITIM. TIGIT was located on the membrane and folded into a functional structure (into, for example, an Ig-like V-type on the membrane, for intercellular signaling).

Reverse transcription-PCR showed that the TIGIT segment was located between 750 and $1000 \mathrm{bp}$, and that TIGIT transcription was detected in mouse organs (Figure 2). The TIGIT sequence was then cloned from pGM-T by PCR (Figure 3). 


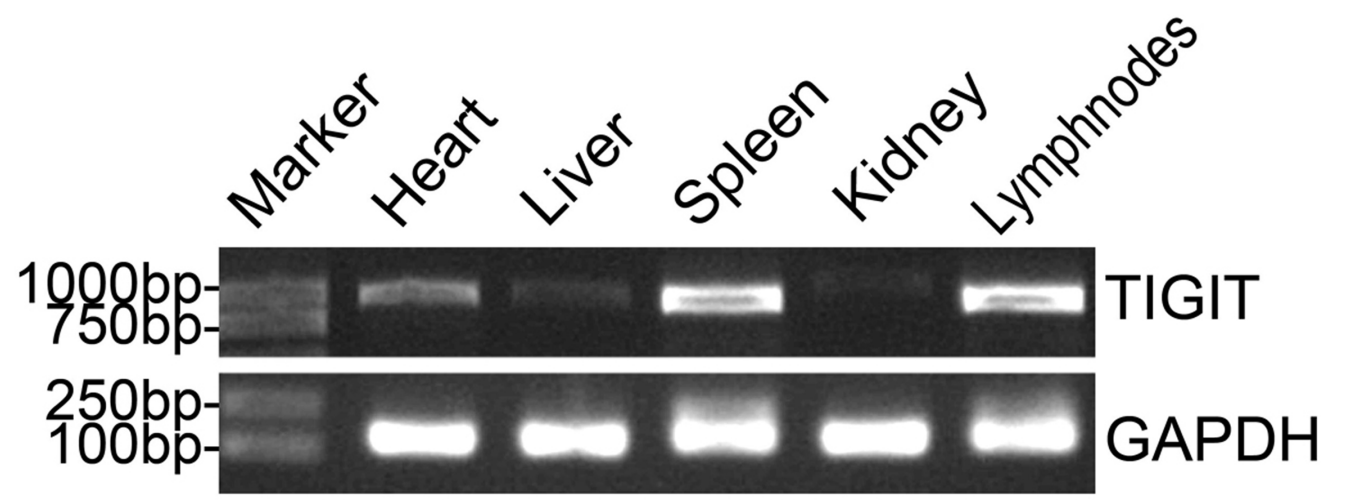

Figure 2. Nucleic acid electrophoresis of TIGIT from mice organs by reverse transcription-PCR. TIGIT was cloned by PCR from the mRNA of each organ. The TIGIT segment (726 bp) was located between 750 and $1000 \mathrm{bp}$. The GADPH segment was used as the control.

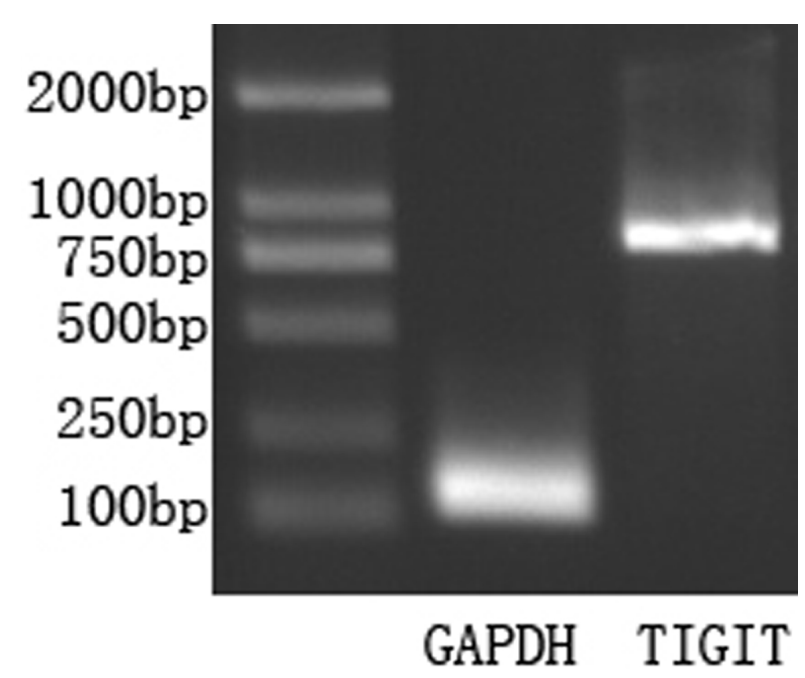

Figure 3. Nucleic acid electrophoresis of TIGIT cloned from pGM-T by PCR. TIGIT cloned from the pGM-T segment was detected on the same position as Figure 2. GADPH was used as the internal control.

\section{Construction of TIGIT-GFP-ADV}

The GFP gene was fused with the TIGIT gene as described in the Materials section (Figure 4). TIGIT-GFP-ADV was constructed in accordance with the planned procedure (Figure 5). TIGIT-GFP from TIGIT-GFP-pENTR1A and GFP from GFP-pENTR1A were double digested with EcoRI and Notl for identification. Nucleic acid electrophoresis showed that the TIGIT-GFP (1473 bp) and GFP (773 bp) segments were cut from the vectors, as expected (Figure 6). Sequencing of both plasmids confirmed the accuracy of the base sequence. The 293A cells infected with TIGITGFP-ADV or GFP-ADV exhibited a cytopathic effect, that is, the cells rounded up and detached from the bottom of the plate, and allowed the formation of viral plaques. Fluorescence microscopic 
observations revealed the presence of GFP in the cytoplasm in the GFP group; on the other hand, GFP was detected on the cytomembrane in the TIGIT-GFP group (Figure 7). Approximately $5 \times 10^{8}$ $293 \mathrm{~A}$ cells were purified to form the viral band (Figure 8 ). According to the titering formula, $6 \mathrm{~mL}$ of $5 \times 10^{10} \mathrm{PFU} / \mathrm{mL}$ TIGIT-GFP-ADV and GFP-ADV (each) was obtained.

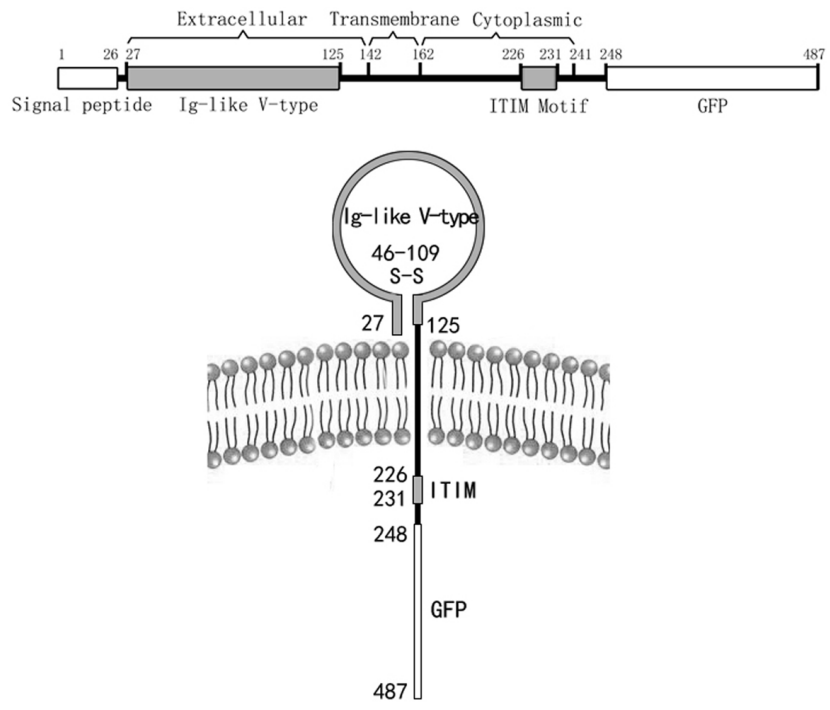

Figure 4. Conception of TIGIT-GFP fusion protein. A GFP was added to TIGIT in order to trace the expression of TIGIT. GFP could be expressed after TIGIT and detected in the same spot as TIGIT on the membrane. This could be confirmed by subsequent testing.
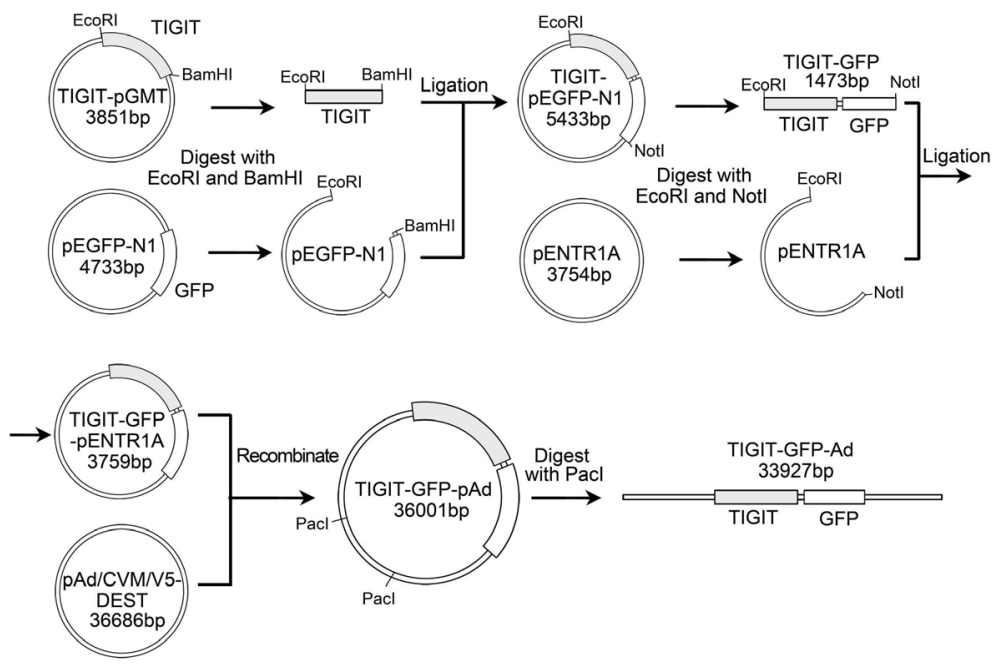

Figure 5. Procedure for construction of TIGIT-GFP-ADV. TIGIT was cut from TIGIT-pGMT and inserted into pEGFP-N1, which contains a GFP segment. The TIGIT-GFP sequence was digested from pEGFP-N1 and inserted into pENTR1A. TIGIT-GFP-pENTR1A and pAd/CVM/V5 DEST were confused to form a TIGIT-GFP-pAd, which was later digested to release TIGIT-GFP-Ad. GFP-Ad was constructed using a similar procedure. 


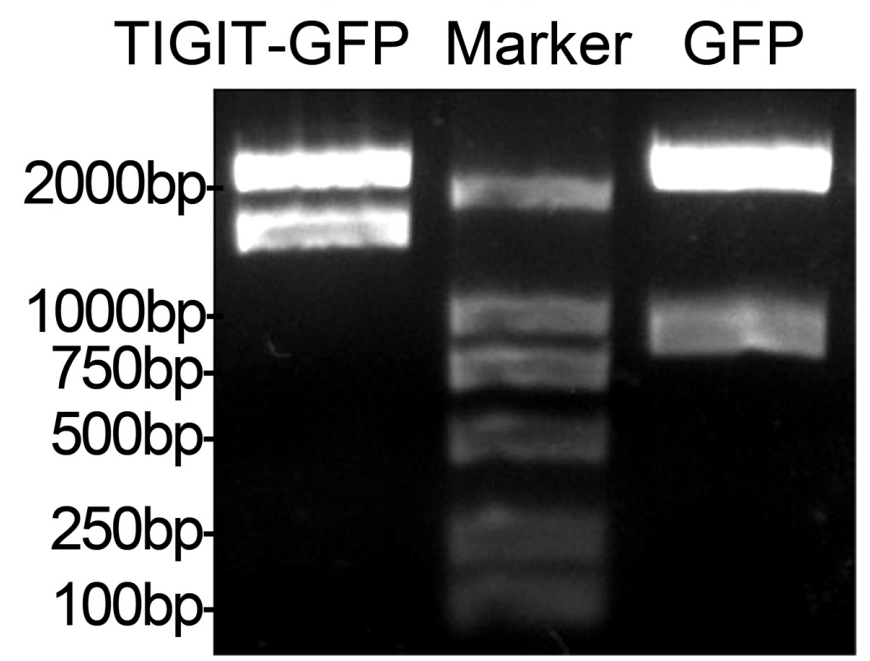

Figure 6. Nucleic acid electrophoresis of TIGIT-GFP and GFP digested with EcoRI and Notl. TIGIT-GFP (1473 bp) and GFP (773 bp) were observed at the expected location.

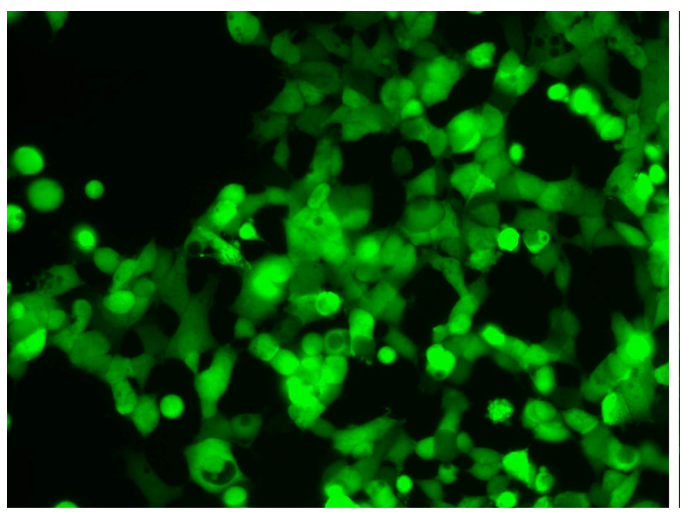

GFP-293A

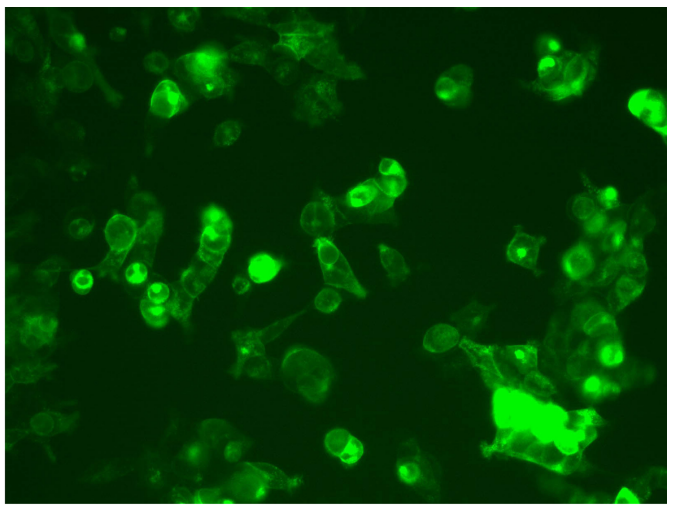

TIGIT-GFP-293A

Figure 7. Detection of GFP in 293A cells infected with GFP-ADV or TIGIT-GFP-ADV, under a fluorescence microscope. GFP was detected in the cytoplasm of the GFP group; in the TIGIT-GFP group, GFP was detected on the membrane.

\section{Characterization of 293A cells infected with TIGIT-GFP-ADV or GFP-ADV}

The expression of TIGIT in 293A cells was examined by flow cytometry. Double positive cells represented the expression of TIGIT and GFP in 293A cells infected with TIGIT-GFP-ADV, and GFP(+) TIGIT(-) cells were detected in 293A cells infected with GFP-ADV. A similar proportion of the two types of cells (22.49 and $25.17 \%$ ) was observed (Figure 9). Immunofluorescence analyses revealed that both TIGIT and GFP were compounded in the cytoplasm and located on the membrane of 293A cells infected with TIGIT-GFP-ADV. The 293A cells infected with GFP-ADV showed the expression of GFP only in the cytoplasm (Figure 10). 


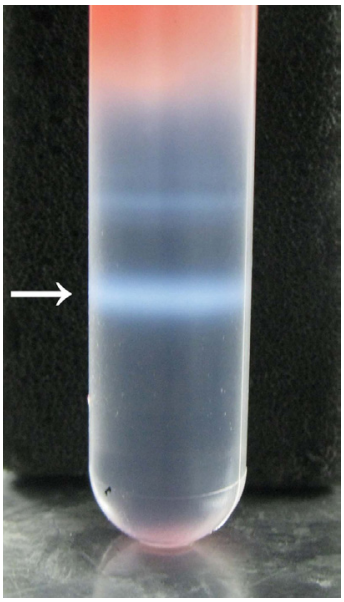

Figure 8. Viral band formed after purification.

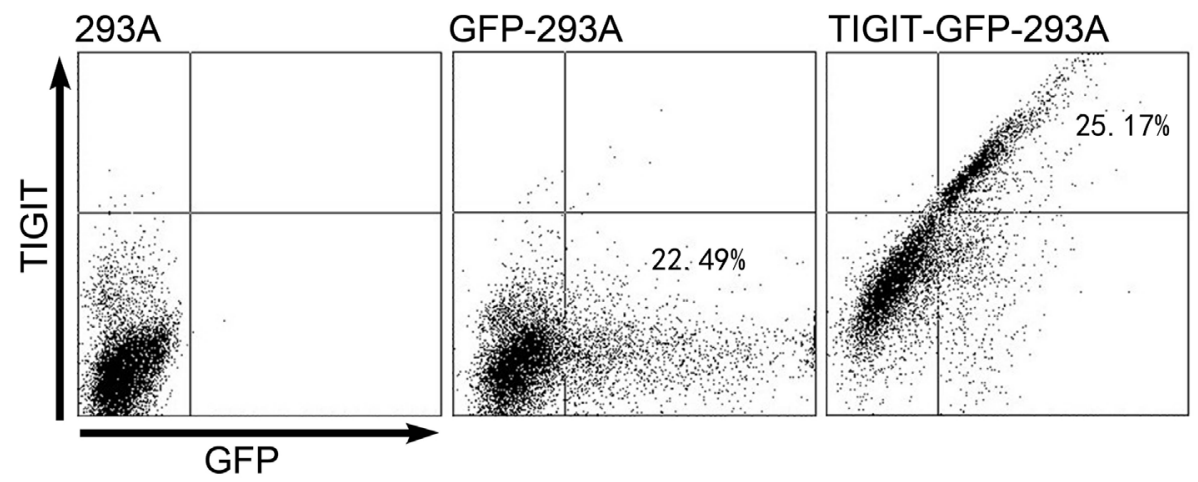

Figure 9. Cell distribution of 293A cells by flow cytometry. GFP(+) cells were detected in the GFP group and GFP(+) TIGIT(+) cells were detected in the TIGIT-GFP group. Each group showed a similar ratio of positive cells.

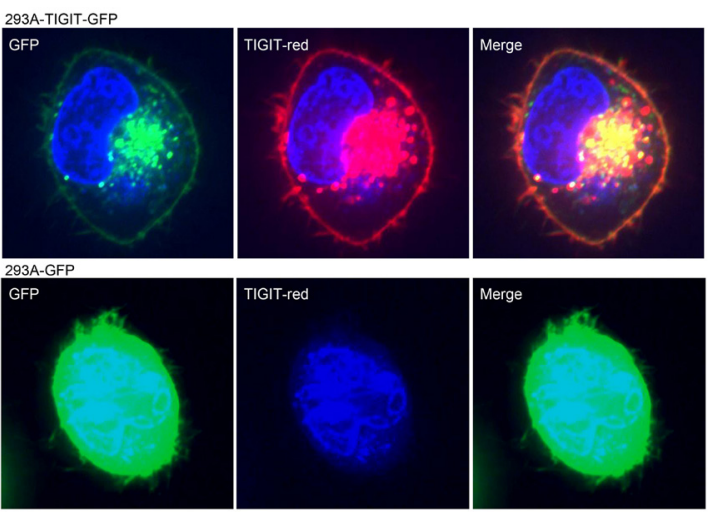

Figure 10. Antibody staining of 293A cells under a confocal microscope. Immunofluorescence studies showed that TIGIT-GFP was expressed and located on the membrane while GFP was expressed in the cytoplasm. 


\section{TIGIT-GFP expression in the liver}

After infection, TIGIT-GFP was found to be highly expressed in the liver on days 1 $(25.53 \%)$ and $3(11.36 \%)$ by flow cytometry analysis; the TIGIT-GFP expression reduced after 5 days $(9.07 \%)$ and 7 days (3.68\%) (Figure 11). Immunofluorescence analyses of the D3 samples showed the expression of GFP and TIGIT on the membrane of liver cells in the TIGIT-GFP group and only GFP in the GFP group (Figure 12).

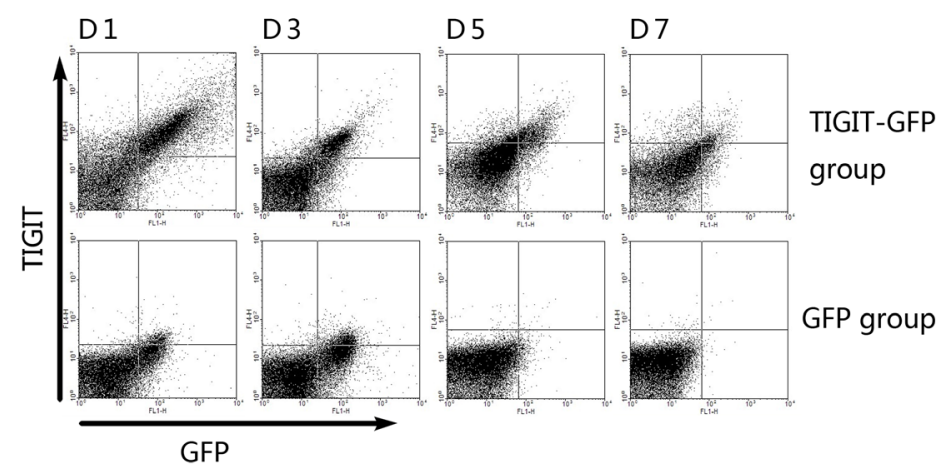

Figure 11. Detection of TIGIT-GFP expression in mouse liver by flowcytometry. TIGIT-GFP or GFP was highly expressed in the liver on days 1 and 3 after infection, and reduced after day 5.
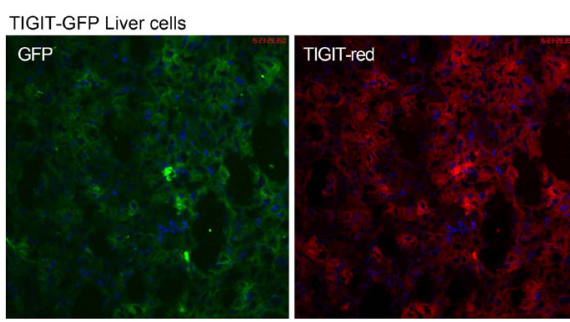

GFP Liver cells

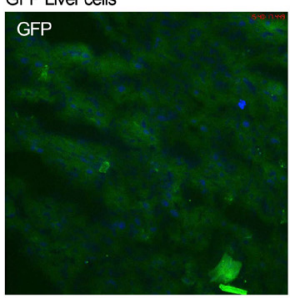

\section{TIGIT-red}

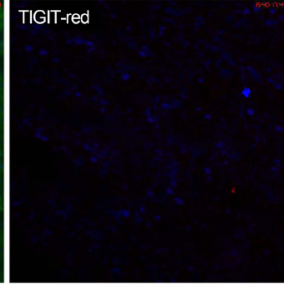

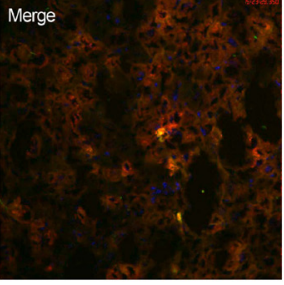

Merge

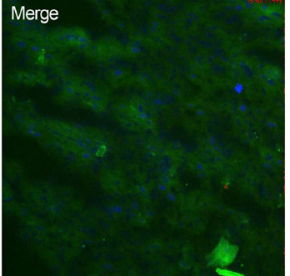

Figure 12. Immunofluorescence analysis of liver cells infected with GFP-ADV or TIGIT-GFP-ADV. GFP and TIGIT were detected on the membrane of liver cells in the TIGIT-GFP group; however, only GFP was detected in the cytoplasm in the GFP group.

\section{DISCUSSION}

Recombinant adenovirus vector systems and gene cloning are important tools for the study of target gene or protein function. The design of gene sequence is particularly important for 
follow-up studies. In this study, the GFP sequence was inserted after the TIGIT sequence in order to synthesize a TIGIT-GFP fusion protein, allowing us to track the fusion protein via GFP under a fluorescence microscope. This facilitates the tracing and positioning of target protein. In addition, the expression of TIGIT need not be traced using a TIGIT-specific antibody during each step of the experiment, as the translation of TIGIT could be assumed in regions where GFP was observed. TIGIT is an immunosuppressive molecule that is currently being researched in studies of immune suppression and tolerance. Preliminary tests indicated that a high expression of TIGIT-GFP in 293A cells could be mediated by the insertion of a recombinant adenovirus vector.

Previous studies have indicated that adenovirus was mainly distributed on the liver in infected animal models (Vrancken et al., 1996; Fujishiro et al., 2005). Similarly, TIGIT-GFP was found to be highly expressed in the mouse liver in this study. This may assist in the treatment of liverrelated autoimmune diseases and liver transplantation in the future (Kren et al., 2002; Tsoulfas et al., 2006). Further research must be conducted to develop an effective gene-based therapeutic strategy for the management of liver-related autoimmune diseases. As recombinant adenovirus could mediate a high expression of target proteins in most mammalian cell lines, it provides a foundation for the study of TIGIT function in vitro and in vivo. Furthermore, the X-GFP adenovirus vector is a useful tool for the analysis of other proteins.

\section{Conflicts of interest}

The authors declare no conflict of interest.

\section{ACKNOWLEDGMENTS}

Research supported by grants provided by the National Basic Research "973" Program of China (\#2009CB522407) and the National Natural Science Foundation of China (\#30972794 and \#81102260). The authors would like to thank Liqun Zuo, Hui Guo, and Su Zhang for expert technical advice, and Ying Xiang, Xia Huang, Chao Li, and Dan Zhang, among others for technical support provided during the course of the research. This study is attributed to the Institute of Organ Transplantation, Tongji Hospital, Tongji Medical College, Huazhong University of Science and Technology, Key Laboratory of Ministry of Health, and Key Laboratory of Ministry of Education.

\section{REFERENCES}

Fujishiro J, Kawana H, Inoue S, Shimizu H, et al. (2005). Efficiency of adenovirus-mediated gene transduction in heart grafts in rats. Transplant Proc. 37: 67-69.

Joller N, Hafler JP, Brynedal B, Kassam N, et al. (2011). Cutting edge: TIGIT has T cell-intrinsic inhibitory functions. J. Immunol. 186: $1338-1342$.

Kren BT, Chowdhury NR, Chowdhury JR and Steer CJ. (2002). Gene therapy as an alternative to liver transplantation. Liver Transpl. 8: 1089-1108.

Levin SD, Taft DW, Brandt CS, Bucher C, et al. (2011). Vstm3 is a member of the CD28 family and an important modulator of T-cell function. Eur. J. Immunol. 41: 902-915.

Lewis TB, Glasgow JN, Harms AS, Standaert DG, et al. (2014). Fiber-modified adenovirus for central nervous system Parkinson's disease gene therapy. Viruses 6: 3293-3310.

Li C, Lu P and Zhang D (1999). Using a GFP-gene fusion technique to study the cell cycle-dependent distribution of calmodulin in living cells. Sci. China C. Life Sci. 42: 517-528.

Lozano E, Dominguez-Villar M, Kuchroo V and Hafler DA (2012). The TIGIT/CD226 axis regulates human T cell function. J. Immunol. 188: 3869-3875. 
Ma CL, Wang GB, Gu RG and Wang F (2010). Construction and characterization of calreticulin-HBsAg fusion gene recombinant adenovirus expression vector. World J. Gastroenterol. 16: 3078-3082.

Stanietsky N, Simic H, Arapovic J, Toporik A, et al. (2009). The interaction of TIGIT with PVR and PVRL2 inhibits human NK cell cytotoxicity. Proc. Natl. Acad. Sci. U. S. A. 106: 17858-17863.

Sun PY, Zhang YL, Jing YM, Zhang XJ, et al. (2011). A comparative analysis of the methods for tittering adenoviruses. J. South Med. Univ. 234-238.

Tsoulfas G, Takahashi Y, Liu D, Yagnik G, et al. (2006). Hydrodynamic plasmid DNA gene therapy model in liver transplantation. J. Surg. Res. 135: 242-249.

Vrancken PM, Perkins AL and Kay MA (1996). Method for multiple portal vein infusions in mice: quantitation of adenovirusmediated hepatic gene transfer. Biotechniques 20: 278-285.

Wang Z, Xiang Q, Wang G, Wang $\mathrm{H}$, et al. (2011). Optimizing expression and purification of an ATP-binding gene gsiA from Escherichia coli k-12 by using GFP fusion. Genet. Mol. Biol. 34: 661-668.

Wang Z, Wang B, Lou J, Yan J, et al. (2014). Mutation in fiber of adenovirus serotype 5 gene therapy vector decreases liver tropism. Int. J. Clin. Exp. Med. 7: 4942-4950.

Wei L, Lu J, Feng L, Li S, et al. (2010). Construction of recombinant adenovirus vector containing a modified gene that codes for human hypoxia-inducible factor-1alpha without oxygen-dependent degradation domain. Plasmid 63: 20-26.

Wong YC, Lin LC, Melo-Silva CR, Smith SA, et al. (2011). Engineering recombinant poxviruses using a compact GFP-blasticidin resistance fusion gene for selection. J. Virol. Methods 171: 295-298.

Yang J, Guo Y, Ren S, Zhou T, et al. (2011). Instantaneous expression aFGF-GFP fusion gene in safflower. Zhongguo Zhong Yao Za Zhi. 36: 281-284.

Yu X, Harden K, Gonzalez LC, Francesco M, et al. (2009). The surface protein TIGIT suppresses T cell activation by promoting the generation of mature immunoregulatory dendritic cells. Nat. Immunol. 10: 48-57. 